\title{
Simultaneity, Chronometrology, and the Two Postulates of Relativity
}

\author{
Steven D. Deines \\ Donatech Corporation, Inc., Fairfield, USA
}

Email address:

sddeines@hotmail.com

\section{To cite this article:}

Steven D. Deines. Simultaneity, Chronometrology, and the Two Postulates of Relativity. International Journal of Applied Mathematics and Theoretical Physics. Vol. 3, No. 3, 2017, pp. 43-49. doi: 10.11648/j.ijamtp.20170303.11

Received: August 21,2016; Accepted: November 26, 2016; Published: May 6, 2017

\begin{abstract}
Einstein gave examples whereby simultaneous events recorded by one inertial observer may not be simultaneous for other inertial observers. This paper eliminates a common misconception. Simultaneous events are confused with separated events occurring at the same coordinate time. Simultaneous events are witnessed by all observers, whether inertial or accelerated, because simultaneous events occur when phenomena collide, merge, overlap, or superimpose into one point at the same instant of time. Chronometric events are separated by a nonzero distance and occur at the same coordinate time of a reference frame. Simultaneous events are witnessed identically by all observers, because a point is still a point with an instantaneous time within any reference frame. Chronometric events occur at identical coordinate times, but are usually not simultaneous, because the distances to convey the information to an observer are usually unequal so that arrival times are different. Einstein's thought experiment to test simultaneity is explained by Newtonian physics without relativity. The mathematics concerning an embellishment of this thought experiment is derived. The contradictory results indicate the two relativity postulates should be revised to establish the correct equations in inertial frames to make identical predictions using the proper transformation.
\end{abstract}

Keywords: Simultaneity, Relativity, Inertial Frames, Speed of Light, Transformation

\section{Introduction}

The laws of physics, especially in relativity, must be stated very precisely to explain natural phenomena within the domain of every inertial reference frame. Einstein developed special and general relativity with two postulates, and he later explained the meaning of his theories with simpler derivations in the form of field equations [1]. Lorrain and Corson [2] stated the fundamental postulate of relativity as: "It is physically impossible to detect the uniform motion of a frame of reference from observations made entirely within that frame". They restated this principle to be emphatically clear, "It means that any experiment gives precisely the same result, whether it is performed in reference frame 1 or in reference frame 2 , or whether it is performed in a standing or in a moving vehicle, as long as there is no acceleration." This is the first special relativity postulate that all physical experimentation performed in inertial frames will be identical upon replication of identical conditions. The author also expects that the experimental output from the same location and perspective will have the same witnessed result when shared simultaneously by multiple inertial observers. This is expected when testing forces that obey Newton's second law. The same force equation applies in all inertial frames, as the derivative of a constant velocity is zero, which means no additional Newtonian force is imparted by the movement of any inertial frame. But, electromagnetic forces do not obey Newton's second law as Lorentz forces are velocity dependent and are different vectors of force depending on the particular inertial frame. The author discussed this in a previous paper [3]. The second postulate of special relativity is the speed of light in a vacuum is the same in all directions for all inertial frames. Serway and Jewett summarized the two postulates of special relativity: "1) The laws of physics must be the same in all inertial reference frames. 2) The speed of light has the same value in all inertial frames, regardless of the observer or velocity of the source emitting the light" [4]. These postulates will be examined thoroughly after clarifying simultaneity in observations. 


\section{Simultaneity Between Inertial Frames}

Einstein $[5, \S 1]$ defined a concept of simultaneity. Later, Einstein [6] explained that whatever is simultaneous for one inertial observer may not be simultaneous when witnessed by another inertial observer initially at the same location, but having a different uniform velocity. He wrote of dual lightning strikes hitting the ground at different locations, A and $\mathrm{B}$, which were witnessed by a ground observer at the midpoint to have been struck simultaneously. Also, a passenger on a perfect train was temporarily at the midpoint, but moved closer toward B while both flashes were traveling toward the passenger, resulting in a nonsimultaneous observation [4, p. 1199-1200].

"Let $\mathrm{M}^{\prime}$ be the mid-point of the distance $\mathrm{A} \rightarrow \mathrm{B}$ on the travelling train. Just when the flashes of lightning occur, this point $\mathrm{M}^{\prime}$ naturally coincides with the point $\mathrm{M}$, but it moves towards the right in the diagram with the velocity $\mathrm{v}$ of the train. If an observer sitting in the position $\mathrm{M}^{\prime}$ in the train did not possess this velocity, then he would remain permanently at $\mathrm{M}$ and the light rays emitted by the flashes of lightning $\mathrm{A}$ and B would reach him simultaneously, i.e. they would meet just where he is situated. Now in reality (considered with reference to the railway embankment), he is hastening towards the beam of light coming from $\mathrm{B}$, whilst he is riding on ahead of the beam of light coming from A. Hence the observer will see the beam of light emitted from B earlier than he will see that emitted from A." [6].

This is expected with Newtonian physics. If any observer was located anywhere on the plane that is a perpendicular bisector of the line between points $\mathrm{A}$ and $\mathrm{B}$ occurring at the same coordinate time, that observer would report a simultaneous occurrence. Any observer located elsewhere perceives nonsimultaneous events, because the distances to convey the phenomena are different, and the arrival times noted by the observer would be different and nonsimultaneous.

This general concept of simultaneity creates confusion concerning the relative nature of simultaneous events and predicting which observer will detect simultaneous events or not. Young, Adams and Chastain [7, p. 863] wrote: "Whether two events at different space points are simultaneous depends on the state of motion of the observer." To add further uncertainty, they wrote [7, p. 864]: "The time interval between two events depends on the frame of reference in which it is measured." The problem is they provided no guidelines how to resolve this difference between observers reporting simultaneous or nonsimultaneous observations from the same phenomena. The core of all experimental physics is obtaining agreement between observers when recording the same phenomena. For example, two observers see a circular plate on a table. One observer was directly above the plate and reported the plate was circular. Another observer saw the same plate as oval. One can transform the circle to an oval by the appropriate cosine projection of the plate, so that one obtains agreement between the two observations with the appropriate geometric projection or transformation. In the same manner, the proper transformation and reference frame should predict where simultaneous observations will be observed based upon nonsimultaneous observations, but no such rule is given.

Two separate interpretations concern simultaneity. The practical definition of simultaneous events is the case that two or more phenomena merge, overlap, superimpose, or coincide at the same point at one instant of time. Technically, an observer is in the neighborhood of this point to perceive simultaneous events, but there is a physical distance between eyes and ears or even a width of an electronic sensor to detect simultaneous events, besides keeping the observer from interfering with the intercepting phenomena. Ignoring these practical observational limitations, an observer can be located conceptually at a point. In this paper, this concept defines simultaneous events. If one had set up perfect stationary clocks at every point in the inertial frame and synchronized them all to a master clock, then one can record the coordinate time at every location that an event occurred. Events with time tags having the same coordinate time can occur at separate points. To clarify this concept from simultaneity, define phenomena with identical time tags as chronometric events. In reality, such events do not have to be detected by an observer. A review of the recorded events and time tags in some log or database is sufficient to establish identical chronometric events. Such equally timed events will appear usually as nonsimultaneous events to an observer, because the distances to communicate or move the chronometric events or phenomena to the observer are different, making the observations appear at separate times.

Any observer, whether inertial or accelerated, should detect the combined events occurring at the same instant at that point as simultaneous. There is no time gap between the arrivals of various phenomena that intercept each other at some time instant. Any transformation of a zero time interval into any other frame of reference must remain a zero time span, because zero is identical in all systems (i.e. 0 days $=0$ years, just as 0 yards $=0$ meters). Furthermore, a point has no length, so a point is still a point in all frames. This is the actual implementation of observed simultaneity. By default, observed simultaneous events are chronometric, because only one time is associated with the combined or merged phenomena [8].

In his example, Einstein did not allow for the reaction time of the eye. The eye cannot detect a time gap shorter than $1 / 24$ second, typical for the frame rate of movies. For example, the passenger could barely detect visually a time gap of 0.2 seconds if the lightning strikes were $29979245.8 \mathrm{~m}$ from the passenger and the train's velocity was $0.1 \mathrm{c}$ using Equations (3) and (4) derived in this paper. Unfortunately, the lightning strikes would be 4.7 Earth radii away from the passenger, making this impossible. High speed electronics are required for the passenger to capture a shorter time gap.

Rindler [9, p. 12-13] agreed with Einstein's 1916 conclusion that the moving passenger sees light from $\mathrm{B}$ before A while the ground observer midway between $\mathrm{A}$ and $\mathrm{B}$ witnesses simultaneous lightning. Einstein allowed the ground observer to have two mirrors at $45^{\circ}$ to the line $\mathrm{AB}$ to witness the dual strikes without turning the observer's head. Sound detection with a microphone would be easier as an equivalent method to record the difference of the thundering heard by the passenger 
and ground observer. There is nothing mysterious or paradoxical about this scenario. All results are consistent entirely within Newtonian physics and do not rely on special relativity, provided the speed of the train is less than the speed of sound so the train passenger could hear and see both events.

The issue is literally the point itself, having no length contraction. All perspective views of a point, even in four dimensions, are identical. All input coincided at the same instant to produce only one sound at the midpoint on the ground. The passenger was not beside the midpoint, but was further down the rail at two different points to recognize that sound from B arrived first and then further down the track to hear sound from $A$. The passenger was not on any point of the bisecting plane of $\mathrm{AB}$ to hear simultaneous events. Rindler defined simultaneity for light as "two events occurring at point $\mathrm{P}$ and $\mathrm{Q}$ of an inertial frame $\mathfrak{I}$ are simultaneous in $\mathfrak{I}$ if and only if light emitted at the two events arrives simultaneously at the midpoint of the segment PQ in $\mathfrak{I} . ”[9$, p. 12]. Although seemingly plausible, Rindler's definition leads to contradictions between inertial frames when assuming the universal speed of light in all directions.

Einstein could have given more attention to the passenger's situation. Suppose the rail was extremely straight and level for tens of kilometers, and the train was over 2 kilometers long with many locomotives pulling it at a steady velocity. Each rail car was equipped with a perfect clock, and all clocks were synchronized to the master ground clock before the train moved. All passenger cars were located in the center section of the train, and freight cars were on the end sections. The freight car $\mathrm{B}^{\prime}$ was near to the locomotives, and the freight car $\mathrm{A}^{\prime}$ was near the caboose. The ground clocks $\mathrm{A}$ and $\mathrm{B}$ are on the ties of the track next to the kilometer marker signs staked 2 kilometers apart. As the train was moving past the ground clocks, the freight car $\mathrm{A}^{\prime}$ was directly above clock $\mathrm{A}$ on the rail tie, and freight car $\mathrm{B}^{\prime}$ was above the ground clock B. One lightning struck both the ground clock and freight car clock chronometrically through points $\mathrm{A}$ and $\mathrm{A}^{\prime}$ in the same neighborhood, and the other lightning stuck both $\mathrm{B}$ and $\mathrm{B}^{\prime}$ concurrently at the same chronometric time. Both clocks $\mathrm{A}^{\prime}$ and $\mathrm{B}^{\prime}$ were shorted out at the same chronometric instant since they were synchronized train clocks. The A and B clocks registered the same time as they were synchronized clocks. The passenger was an avid tourist and was recording very high speed video and sound to preserve the entire special trip. The video was also recording the time stamped from the passenger car clock. As Einstein and Rindler predicted, the high speed recording showed the lightning and thunder from the strikes were both nonsimultaneous. The engineer stopped the train at the next depot to inspect the damage for safety reasons, which gave plenty of time for the curious passenger to measure the distance between the passenger's seat and the damage marked on rail cars $\mathrm{A}^{\prime}$ and $\mathrm{B}^{\prime}$. The passenger noted the stopped clocks at $\mathrm{A}^{\prime}$ and $\mathrm{B}^{\prime}$ were identically the same, and the passenger's seat was at the midpoint of the two kilometer distance of $\mathrm{A}^{\prime} \mathrm{B}^{\prime}$ as measured by the charred marks on the rail cars $A^{\prime}$ and $B^{\prime}$. There was no wind to affect the sound speed, and the passenger asked the engineer the train's velocity, $v$, when the lightning struck. With this information and knowing the speeds of sound, s, and light, c, the passenger calculated the effective one-way speeds of sound and light in the inertial frame of the moving train.

The embellishment of Einstein's dual lightning events does not alter his prediction that the passenger $\mathrm{P}$ recorded nonsimultaneous events. The second postulate of relativity from Einstein is the speed of light is the same in all directions in all inertial, nongravitated reference frames. Even if the velocity of the train causes length contraction of the meter compared to the meter of the ground frame, the lengths of $\mathrm{A}^{\prime} \mathrm{P}$ and $\mathrm{B}^{\prime} \mathrm{P}$ are identical. With the speed of light being the same from $\mathrm{A}^{\prime}$ to $\mathrm{P}$ or in reverse direction of $\mathrm{B}^{\prime}$ to $\mathrm{P}$, the passenger should still detect simultaneous events as defined by Rindler. Both Einstein and Rindler emphasized the passenger would not observe simultaneous events in this scenario. The passenger is fixed at the midpoint between the dual lightning strikes with all the information permanently recorded in the inertial frame of the moving train. The passenger can still measure the distances between $\mathrm{A}^{\prime} \mathrm{P}$ and $\mathrm{B}^{\prime} \mathrm{P}$ while the train moves, and the midpoint of $\mathrm{A}^{\prime} \mathrm{B}^{\prime}$ is where the passenger and the recording equipment were located. Rindler's definition of simultaneity requires that the arriving light from the lightning strikes be simultaneous at the passenger's seat, yet Rindler agreed with Einstein that the passenger and equipment observed nonsimultaneous events. As the moving railcar qualifies as an inertial frame, the second postulate of relativity requires the passenger at midpoint to observe simultaneous events as the distances $\mathrm{A}^{\prime} \mathrm{P}$ and $\mathrm{B}^{\prime} \mathrm{P}$ are equal and the speed of light is a universal constant.

Einstein's point of this theoretical case was to make the casual reader understand that simultaneity does not apply to separated phenomena that have the same coordinate time, which are chronometric events under the author's definition. The author agrees with Einstein's statement of nonsimultaneous observations by the train passenger, because the passenger observed both lightning and thunder at locations closer to B than A even though the dual lightning strikes are chronometric events. If the passenger was on the bisecting plane instantly that light or sound reached the passenger, then the passenger must observe simultaneous events, too. Chronometric events are separated events having the same coordinate time of occurrence, but such chronometric events are not usually simultaneous, which require merged events at one point at the same single instant of time.

Both postulates of relativity are dubious in Einstein's train scenario. The inertial ground frame is equivalent to an absolutely stationary frame, while the passenger's frame is a moving inertial frame. The ground observer and passenger do not experience the same phenomena. Both verified chronometric lightning events. The midpoint ground observer recorded simultaneous lightning and thundering, but the passenger did not. The ground observer had no wind, but the passenger noticed an effective wind through an open window when moving. The passenger's calculations that allowed vector addition of sound velocity agreed with the observed 
time interval between the thunder claps. Replacing the sound speed with light speed in the passenger's analysis indicates light has different speeds in different directions, which explains the nonsimultaneous observations from lightning. Thus, both relativity postulates are at odds with Einstein's train scenario.

\section{Velocity Measurements in a Moving Inertial Frame}

Many interesting relationships are obtained from this

$$
\begin{gathered}
L+v \frac{L}{c}+v \frac{L v / c}{c}+\cdots+v \frac{L v^{n-1} / c^{n-1}}{c}=L+L \frac{v}{c}+L \frac{v^{2}}{c^{2}}+\cdots+L \frac{v^{n}}{c^{n}}=\frac{L\left(1-v^{n} / c^{n}\right)}{1-v / c} \\
L_{A P}=\lim _{n \rightarrow \infty} \frac{L\left(1-v^{n} / c^{n}\right)}{1-v / c}=\frac{L}{1-v / c}=L\left(1+\frac{v / c}{1-\frac{v}{c}}\right)
\end{gathered}
$$

From the ground point $\mathrm{B}$, the light travels a shorter distance than $\mathrm{L}$ of $1 \mathrm{~km}$ as the passenger moves toward it. Shorten the total distance by removing increments of distance $\xi(i)$ instead of adding. The time from $\mathrm{B}$ to the passenger is scenario. The light from A in the ground frame moves toward the passenger, who is traveling to $\mathrm{B}$ at the uniform velocity $\mathrm{v}$. When that light travels a distance L (or $1 \mathrm{~km}$ ) from A over the time $\mathrm{L} / \mathrm{c}$, the passenger is a further distance of $\xi(1)=\mathrm{v} \mathrm{x}$ $\mathrm{L} / \mathrm{c}$ along the track. When that light travels the extra distance $\xi(1)$, the passenger has moved a further distance $\xi(2)$ over the same time interval of $\xi(1) / \mathrm{c}$. Over $\mathrm{n}$ repetitions of this, the passenger has moved a distance of $\mathrm{L}+\xi(1)+\xi(2)+\ldots+$ $\xi(\mathrm{n})$ where $\xi(1)=\mathrm{v} \times(\mathrm{L} / \mathrm{c})$ and $\xi(\mathrm{i}+1)=\mathrm{v} \times \xi(\mathrm{i}) / \mathrm{c}$. Substitute the individual terms, and the series is $[10,11]$ :

$$
\begin{gathered}
\mathrm{L}\left(1-\frac{\mathrm{v}}{\mathrm{c}}-\frac{\mathrm{v}^{2}}{\mathrm{c}^{2}}-\cdots\right)=\mathrm{L}\left(2-1-\frac{\mathrm{v}}{\mathrm{c}}-\frac{\mathrm{v}^{2}}{\mathrm{c}^{2}}-\cdots\right)=\mathrm{L}\left(2-\left[1-\frac{\mathrm{v}}{\mathrm{c}}-\frac{\mathrm{v}^{2}}{\mathrm{c}^{2}}-\cdots\right]\right)= \\
\mathrm{L}\left(2-\frac{1}{1-\frac{\mathrm{v}}{\mathrm{c}}}\right)=\mathrm{L}\left(\frac{1-2 \mathrm{v} / \mathrm{c}}{1-\mathrm{v} / \mathrm{c}}\right)=\mathrm{L}_{\mathrm{BP}}=\mathrm{L}\left(1-\frac{\mathrm{v} / \mathrm{c}}{1-\frac{\mathrm{v}}{\mathrm{c}}}\right)
\end{gathered}
$$

If one considers the speed of sound with no wind, the arguments remain the same, and the resulting formulas are the same form as (1) and (2) except replace c with $s$, the speed of sound, due to the thundering. Equations (1) and (2) can be converted into a time interval by dividing by the velocity of light or sound as appropriate.

$$
\begin{gathered}
\Delta \mathrm{T}_{\mathrm{AP}}=\frac{\frac{\mathrm{L}}{\mathrm{c}}}{1-\mathrm{v} / \mathrm{c}}=\mathrm{T}\left(1+\frac{\mathrm{v} / \mathrm{c}}{1-\frac{\mathrm{v}}{\mathrm{c}}}\right) \text { where } \mathrm{T}=\frac{\mathrm{L}}{\mathrm{c}} \text { for } \mathrm{L} \text { of } 1 \mathrm{~km}, \text { and } \\
\Delta \mathrm{T}_{\mathrm{BP}}=\frac{\mathrm{L}}{\mathrm{c}}\left(\frac{1-2 \mathrm{v} / \mathrm{c}}{1-\mathrm{v} / \mathrm{c}}\right)=\mathrm{T}\left(1-\frac{\mathrm{v} / \mathrm{c}}{1-\frac{\mathrm{v}}{\mathrm{c}}}\right) .
\end{gathered}
$$

Note that $\mathrm{v}<\mathrm{c}$ or $\mathrm{v}<\mathrm{s}$ as appropriate to allow light or sound from A to reach the passenger. For sound calculations, replace $\mathrm{c}$ with $\mathrm{s}$ in (3) and (4). Multiply by the velocity of the train $\mathrm{v}$ against the time intervals predicted by (3) and (4) to get the two locations that the passenger will hear the thunder beyond the midpoint ground observer in the direction of $\mathrm{B}$. Add two ground observers beside the rail at these locations. The second ground observer closer to the midpoint technically sees the light emanated off the passenger through the passenger car window and detects the sound from B simultaneously. Concurrently, the passenger hears the thunder from B and sees the second ground observer. This is repeated later when the third ground observer simultaneously sees the passenger pass the location and hears the sound from A. Also, the passenger simultaneously sees the third ground observer and hears the thundering from A. The two additional ground observers confirm in the ground frame that the passenger heard separate thundering at two separate locations at these predicted points and times, which is the same result recorded by the passenger still seated in the train's frame of reference. The three ground observers eventually report that the thundering from $\mathrm{A}$ and $\mathrm{B}$ were chronometric when they inspect the stopped clocks at A and B. Only the midpoint observer reported simultaneous lightning and thundering after the ground strikes at $\mathrm{A}$ and $\mathrm{B}$. The other two ground observers reported nonsimultaneous events, because these two ground observers were not located equidistant between A and B.

The seated passenger was stationary in the train's frame of reference. When the train stopped at the next depot, the passenger measured the distances from the passenger's seat to the two lightning strikes burned on the two railcars, which were equal lengths $L$ of 1 kilometer. Using the time intervals recorded between the stopped clocks at rail cars $\mathrm{A}^{\prime}$ and $\mathrm{B}^{\prime}$ versus the times recorded by the passenger's equipment, the passenger calculates the effective speeds of light, c, (or sound, s) from $\mathrm{A}^{\prime}$ and $\mathrm{B}^{\prime}$ are: 


$$
\begin{gathered}
\mathrm{C}_{\mathrm{AP}}=\frac{\mathrm{L}}{\frac{\mathrm{L}}{\mathrm{c}}\left(1+\frac{\mathrm{v}}{\mathrm{c}}\right)}=\mathrm{c}\left(\frac{1-\frac{\mathrm{v}}{\mathrm{c}}}{\mathrm{c}}\right)=\mathrm{c}\left(\frac{\mathrm{c}-\mathrm{v}}{\mathrm{c}}\right)=\mathrm{c}-\mathrm{v} \\
\mathrm{C}_{\mathrm{BP}}=\frac{\mathrm{L}}{\frac{\mathrm{L}}{\mathrm{c}}\left(1-\frac{\frac{\mathrm{v}}{\mathrm{c}}}{1-\frac{\mathrm{v}}{\mathrm{c}}}\right)}=\mathrm{c}\left(\frac{1-\frac{\mathrm{v}}{\mathrm{c}}}{1-\frac{2 \mathrm{v}}{\mathrm{c}}}\right)=\mathrm{c}\left(1+\frac{\frac{\mathrm{v}}{\mathrm{c}}}{1-\frac{2 \mathrm{v}}{\mathrm{c}}}\right) \approx \mathrm{c}\left(1+\frac{\frac{\mathrm{v}}{\mathrm{c}}}{1}\right)=\mathrm{c}+\mathrm{v} \text { if } \mathrm{v}<<\mathrm{c}
\end{gathered}
$$

Interchanging $\mathrm{s}$ and $\mathrm{c}$ in (5) and (6), these results show the effective speeds of light and sound in one direction are affected by the speed of the inertial frame relative to an absolutely stationary frame. This may seem paradoxical, because the many laboratory measurements for the speed of light have improved precision to less than a meter per second, and the overall round trip light speed has always remained the same, regardless of Earth's orbital velocity of nearly $29,800 \mathrm{~m} / \mathrm{s}$ revolving about the Sun.

Let the passenger in the train measure the speed of light through the air using a laser pulse aimed at a mirror on the opposite wall of the passenger car so that it reflects back to the source. Light speed will be adjusted for the air's index of refraction to get the equivalent speed $\mathrm{c}$ in a vacuum. The precise clock onboard the passenger car measures the total time interval of transmission from the laser by the round trip over the length of the car. In terms of the ground (or absolutely stationary) frame, the parallel light pulse going through the air toward the mirror of the moving passenger car would traverse a longer distance $\mathrm{L}_{\rightarrow}$ than the returning light moving antiparallel of the moving car for its distance of $\mathrm{L}_{\leftarrow}$. The derivation of the two lengths has been derived in Equations (1) and (2), respectively. The sum is:

$$
\mathrm{L}_{\rightarrow}+\mathrm{L}_{\leftarrow}=\mathrm{L}\left(\frac{1}{1-\mathrm{v} / \mathrm{c}}\right)+\mathrm{L}\left(\frac{1-2 \mathrm{v} / \mathrm{c}}{1-\mathrm{v} / \mathrm{c}}\right)=\mathrm{L}\left(\frac{2-2 \mathrm{v} / \mathrm{c}}{1-\mathrm{v} / \mathrm{c}}\right)=2 \mathrm{~L} .
$$

The surprising result is that the passenger measures the total length of $2 \mathrm{~L}$ during the transmission, regardless of the speed of the train as long as $\mathrm{v}<\mathrm{c}$ to allow the emitted light to reach the mirror. If the train was stationary to the ground, the passenger measures the speed of light $\mathrm{c}$ in the absolutely stationary frame. Divide the total distance by c to get $2 \times \Delta \mathrm{T}$, where $\Delta \mathrm{T}=\mathrm{L} / \mathrm{c}$. In repeated measurements of the total time for a round trip light test, the passenger will get the same result of $2 \Delta \mathrm{T}$, regardless of the actual speed $\mathrm{v}$ of the train relative to an absolutely stationary frame.

$$
\Delta \mathrm{T}_{\rightarrow}+\Delta \mathrm{T}_{\leftarrow}=\Delta \mathrm{T}\left(\frac{1}{1-\mathrm{v} / \mathrm{c}}\right)+\Delta \mathrm{T}\left(\frac{1-2 \mathrm{v} / \mathrm{c}}{1-\mathrm{v} / \mathrm{c}}\right)=\Delta \mathrm{T}\left(\frac{2-2 \mathrm{v} / \mathrm{c}}{1-\mathrm{v} / \mathrm{c}}\right)=2 \Delta \mathrm{T},
$$

where $\Delta \mathrm{T}_{\rightarrow}=\Delta \mathrm{T}\left(1+\frac{\mathrm{v} / \mathrm{c}}{1-\mathrm{v} / \mathrm{c}}\right)$ and $\Delta \mathrm{T}_{\leftarrow}=\Delta \mathrm{T}\left(1-\frac{\mathrm{v} / \mathrm{c}}{1-\mathrm{v} / \mathrm{c}}\right)$. In Einstein's famous 1905 article that established special relativity [5], he wrote that remote coordinate clocks could be synchronized by adding half of the round trip time interval to the broadcast time from the master clock. He was considering the light off the master clock face or a radio broadcast of the master clock time. The problem is that the Einstein synchronization procedure requires the reference frame to be absolutely stationary, where $\mathrm{v}$ must be zero for $\Delta \mathrm{T}_{\rightarrow}=$ $\Delta \mathrm{T}_{\leftarrow}=\Delta \mathrm{T}$. However, in all cases when $\mathrm{v}>0, \Delta \mathrm{T}_{\rightarrow}>\Delta \mathrm{T}>$ $\Delta \mathrm{T}_{\leftarrow}$ as the general case. This underscores the issue of synchronizing coordinate clocks to a chosen master clock if the observer in a moving inertial frame has no means of determining the moving frame's velocity relative to an absolutely stationary frame of reference when using inertial measuring devices. To synchronize remote clocks to a master clock, the velocity of the moving frame is necessary to define the elapsed time of transmission according to (3) and (4) to add at the broadcast time and ensure the remote clocks are synchronized to the master clock. This specific issue is beyond the scope of this paper and will be addressed in a subsequent paper.

The passenger predicts the first ground observer will hear simultaneous thundering from A and B using the different speeds of sound from opposite directions relative to the moving inertial frame fixed on the train. Initially, the ground observer at the midpoint was beside the passenger in the railcar at the chronometric time that lightning struck both $\mathrm{A}$ and B. The ground observer has an effective velocity - $v$ away from the passenger, the sound from B has $\mathrm{s}+\mathrm{v}$ speed by (6), and the sound from $A$ by (5) has $s-v$ speed. The distance between $A^{\prime}$ and the ground observer is $L$, which is the same between $\mathrm{B}^{\prime}$ and the ground observer. The mutual velocity between the approaching sound from $\mathrm{A}$ to the ground observer in opposite directions is $(\mathrm{s}-\mathrm{v})-(-\mathrm{v})=\mathrm{s}_{\mathrm{A}}$. The velocity between the overtaking sound from $B$ to the ground observer in the same direction is $(\mathrm{s}+\mathrm{v})+(-\mathrm{v})=\mathrm{s}_{\mathrm{B}}$. Calculating transmission times between $\mathrm{A}^{\prime}$ and the first (or midpoint) ground observer and between $\mathrm{B}^{\prime}$ and that observer using unequal sound speeds, the passenger predicts the ground observer hears simultaneous thundering obtained from vector addition. The same substitutions for light would also apply. In either the ground or train frames, the midpoint observer detected simultaneous events while the passenger detected nonsimultaneous events from the same chronometric lightning strikes. Thus, inertial frames do not preserve velocities when dealing with physical speeds measured in each inertial frame, and the speed of light is not a universal constant in all directions in Einstein's simultaneity experiment between two inertial frames,

\section{Conclusion}

Einstein [6] described that events may be reported as simultaneous by some inertial observers and nonsimultaneous by other inertial observers. He gave the 
example of dual lightning strikes, which he termed as "simultaneous", on the railroad where one stationary ground observer was located midpoint between the two lightning strikes and a passenger on a uniformly moving train was temporarily at the midpoint. To remove the apparent paradox, definitions are given for simultaneous and chronometric events. Simultaneous events occur where transported phenomena combine at the same point at the same instant of time. This concept is more definitive and more restrictive than the past definitions that simultaneous events happen, exist, or occur at the same coordinate time. By this new definition, all observers (whether stationary, moving uniformly, or accelerated relative to the point of interest) will report the events as simultaneous. A zero distance and a zero time interval between such combined events remain zero when transformed into any observer's reference frame.

Chronometric events are phenomena that exist at the same coordinate time, regardless of location, as recorded by an array of perfectly synchronized clocks located throughout the inertial reference frame. Such clocks maintain coordinate time when slaved to a perfect master clock. Technically, chronometric events do not have to be observed directly, as long as the time tag is recorded with each event of interest at a location for later retrieval. Virtually all chronometric events from different locations are viewed as nonsimultaneous by observers, because the distance is different to transport the phenomena of each originating event to the observer, thereby making the arrival times different. Also, simultaneous events are usually not chronometric at the originating locations, such as simultaneously observed novae erupted at different coordinate times and locations due to the different distances between Earth and the novae.

Without changing Einstein's conditions, his train scenario is embellished with additional ground observers, no wind to have the same speed for sound in all directions of the ground frame, precise recording and timing equipment, and an extra long train that was hit by the dual chronometric lightning at equidistant points $\mathrm{A}^{\prime}$ and $\mathrm{B}^{\prime}$ from the passenger and at ground points $\mathrm{A}$ and $\mathrm{B}$. The only ground observer that recorded simultaneous lightning and thunder was midway between points $\mathrm{A}$ and $\mathrm{B}$. The length that light or sound travels from $\mathrm{A}$ to the receding passenger is longer than light or sound traverses from B to the approaching passenger, as expected. Added ground observers standing beside the rail at the passenger's locations detecting light or sound from A or B confirm the nonsimultaneous reception as reported by the passenger. The freight cars with synchronized clocks at $\mathrm{A}^{\prime}$ and $\mathrm{B}^{\prime}$ were collocated above the pair of synchronized ground clocks at A and B when lightning struck all 4 clocks chronometrically. The passenger checks the charred markings on the train at $\mathrm{A}^{\prime}$ and $\mathrm{B}^{\prime}$ and the clocks in the freight cars and concluded the times and lengths to the passenger's seat are the same. With the universal speed of light or sound in the ground frame, the mathematics predicts the passenger would report simultaneous lightning or thunder by Rindler's definition, but the passenger did not as stated by Einstein and Rindler. The conclusion is that the one-way speeds of light and sound are different in different directions within the passenger's reference frame, which would explain the observed nonsimiultaneity in the passenger's frame. In the absolutely stationary frame (i.e. the ground frame), the passenger's nonsimultaneity is because the light and sound travel different distances $\mathrm{A}^{\prime} \mathrm{P}$ versus $\mathrm{B}^{\prime} \mathrm{P}$ for the passenger to observe at different times, despite the uniform velocity of light or sound within the stationary frame.

This is a true conflict with the first fundamental postulate of relativity. The chronometric, dual lightning event resulted in simultaneous observations of lightning flashes and thunder clasps for the midpoint ground observer. In the same identical event in a moving inertial frame, the train passenger recorded nonsimultaneous events when lightning struck at the same coordinates times and same distances away from the passenger. No force exists between the ground observers and passenger, so no force affects the observations collected by any observer. However, when phenomena move with a velocity, one must account for the mutual velocity between inertial frames to get the correct equations of motion for both reference frames. In the embellished scenario, the speed of sound, s, is constant in the ground frame since there is no wind relative to the ground. The passenger witnesses an effective wind antiparallel to the train's speed, v, through an open window. The passenger verifies the nonsimultaneous thunder by calculating the speed of sound from A as s-v and the speed of sound from B as $\mathrm{s}+\mathrm{v}$ (provided $\mathrm{v}<<\mathrm{s}$ ). The Galilean transformation successfully accounts for the differences in the speed of sound as a function of direction if one includes the mutual velocity between the two inertial frames. Thus, the velocity equations that predict timed observations of speeding phenomena are not the same general form between inertial frames, making a necessary update to the first postulate of relativity. One suggested redefinition of the first postulate of relativity is all observed forces that obey Newton's second law give the same experimental results when tested in inertial frames.

This embellished scenario also conflicts with the second postulate of relativity concerning the constancy of light speed in all directions. According to Rindler's definition of simultaneity [9], the passenger must observe simultaneous lightning strikes as the passenger is midway between the two chronometric timed events (i.e. same coordinate times) in the train's inertial frame. However, both Einstein and Rindler correctly state that the midpoint ground observer will report simultaneous lightning while the passenger will not. The resolution of this paradox is the velocity of light obeys vector addition similar to the velocity of sound. This is shown in the derivation that light from $\mathrm{A}$ travels a longer distance to overtake the moving passenger compared to light from B that has a shorter traverse as the passenger approaches. The reason that light appears to have the same speed in all directions in an inertial frame is that all precise light speed measurements are done in a round-trip course that effectively uses one precise clock to measure the time interval when the reflected light beam arrives after traveling a length of $2 \mathrm{~L}$. In an absolutely stationary inertial frame (e.g. the flat-Earth 
ground frame of Einstein's example), a reflected light beam travels a total distance of $2 \mathrm{~L}$ over the course of length $\mathrm{L}$ between mirrors. When placing the experiment in the passenger railcar, the light beam travels a longer distance moving parallel to the train's velocity to reach the reflecting mirror and a shorter distance as light moves antiparallel of the train's velocity back to the origin relative to the stationary reference frame, but the total distance is still $2 \mathrm{~L}$ in either the stationary or moving inertial frame. As long as a velocity $\mathrm{v}<$ $\mathrm{c}$ allows light to reach the reflecting mirror, the total distance of $2 \mathrm{~L}$ is the same in all roundtrip experiments, and the total time of a round-trip light speed test is $2 \Delta \mathrm{T}=2 \mathrm{~L} / \mathrm{c}$ as in an absolutely stationary frame. In the moving inertial passenger frame, the passenger's nonsimultaneous observations can only be explained if there are different speeds of light or sound from the separate locations $\mathrm{A}^{\prime}$ and $\mathrm{B}^{\prime}$, because the dual lightning and thundering are marked on the train at identical distances from the passenger's seat. If the speed of light in one direction is different between moving inertial frames, this basic tenant of relativity is contradicted. It appears the Galilean transformation is correct compared to the Lorentz transformation, which the latter requires a universal speed for light in all directions.

\section{References}

[1] Einstein, A., The Meaning of Relativity, $5^{\text {th }}$ ed., MJF Books, (1954).
[2] Lorrain, P. \& Corson, D. A., Electromagnetic Fields and Waves, W. H. Freeman and Company, $2^{\text {nd }}$ ed., (1970), p. 461.

[3] Deines, S. D. "Functional Basic Units of Physics and Reference Frames that Preserve Maxwell's Equations", IJAMTP, 2 (1), (2016).

[4] Serway, R. A. and Jewett Jr., J. W., Physics for Scientists and Engineers, Cengage Learning (2014), p. 1196-1198.

[5] Einstein, A., "On the Electrodynamics of Moving Bodies" Ann. Phys., 17, p. 549-560, (1905) [translated from Perrett, W., and Jeffery, G. B., in The Principle of Relativity: ..., Metheuen, London, 1923].

[6] Einstein, A., Relativity: the Special and the General Theory, (1916) reprinted Three Rivers Press, Random House Inc., 15th ed. (1961).

[7] Young, H. D., Adams, P. W. and Chastain, R. J., Sears and Zemansky's College Physics, Pearson, (2016), 10th ed.

[8] Deines, S. D., “Timing in Simultaneity, Einstein's Test Scenario, and Precise Clock Synchronization”, IJAMTP, 3 (1), (2017).

[9] Rindler, W., Special Relativity, University Mathematical Texts, Interscience Publishers Inc. (1966) reprinted by John Wiley \& Sons (1969).

[10] Deines, S. D., "Vector Addition of Light Speed Versus the Hafele-Keating Time Dilation Test", to be published in IJAMTP (2017).

[11] Deines, S. D., "Dichotomy between Length Contraction and Null Results from All Interferometer Experiments", to be published in IJAMTP (2017). 